\title{
Investigation of the effect of a bumpy base on granular segregation and transport properties under vertical vibration
}

\author{
C. C. Liao, ${ }^{1,2}$ M. L. Hunt, ${ }^{2}$ S. S. Hsiau, ${ }^{1, \text { a) }}$ and S. H. Lu ${ }^{1}$ \\ ${ }^{1}$ Department of Mechanical Engineering, National Central University, No. 300, \\ Jhongda Road, Jhongli 32001, Taiwan \\ ${ }^{2}$ Caltech, Division of Engineering and Applied Science, Pasadena, California 91125, USA
}

(Received 11 April 2014; accepted 2 July 2014; published online 23 July 2014)

\begin{abstract}
This study experimentally investigates the effect of a bumpy base on the Brazil-nut phenomenon in a vertically vibrated granular bed. The rise dynamics of an intruder is determined by the particle tracking method. The results indicate that the rise time increases with an increase in the base roughness, and the variation of the rise time with different base factors is more pronounced with smaller vibration acceleration and higher vibration frequency. A theoretical model is employed to measure the penetration length of the intruder and the drag force between the intruder and the immersed beads. The penetration length is reduced and the drag force is enhanced with surface roughness of the base. Additionally, the transport properties of the vibrated glass beads are also measured and discussed. With greater base roughness, the strength of the diffusive and convective motion is reduced leading to a weaker Brazil-nut effect. @ 2014 AIP Publishing LLC. [http://dx.doi.org/10.1063/1.4890363]
\end{abstract}

\section{INTRODUCTION}

Granular materials, collections of discrete solid particles, are usually observed in daily life and widely found in many industries. Segregation of granular materials is a critical issue in many industrial processes, such as pharmaceutical products and fluidized bed reactors, and is an interesting phenomenon that may occur in vibrated granular beds. Granular segregation may be impacted due to variations in grains size, density, shape, friction coefficient, and restitution coefficient. The wellknown "Brazil nut problem," in which a large particle (the so-called intruder) rises relative to the surrounding smaller-sized granular material when vertically vibrated, has been studied extensively by experiments, theoretical models, and numerical simulations. ${ }^{1-17}$ In a vibrating granular bed, the segregation may also be influenced by the vibration conditions, bed height, ambient pressure, humidity, size ratio, density ratio, restitution coefficient, and the friction coefficient of the particles. ${ }^{8,9,12,16}$ Granule segregation in a vertically-vibrated granular bed is highly complicated and much remains unknown.

Understanding the rising of an intruder that is immersed in a granular material is a challenge for granular flow research. Prior studies have shown that the granular material may undergo a transition from a dense (solid-like) state to a dilute (liquid-like) state as sufficient external energy is applied to the granular system. ${ }^{10}$ During the expansion of the granular bed, voids form between particles allowing particles to reorganize and the smaller particles to fall through the voids to the bottom of the bed, thus resulting in size segregation. ${ }^{4,10}$ Duran et al. ${ }^{1}$ indicated that larger disks rise upward continuously in small steps via the arching effect, in which the intruder is supported by the surrounding network of smaller particles; smaller particles, however, may only climb up intermittently when amplitude of the excitation is strong enough. Cooke et al. ${ }^{2}$ reported their

\footnotetext{
a) Author to whom correspondence should be addressed. Electronic mail: sshsiau@cc.ncu.edu.tw. Tel.: +886 34267341. Fax: +88634254501 .
} 
experimental results on the rise dynamics of an intruder in a 2D vertically-vibrating bed and found that the segregation rate is enhanced as the peak acceleration and size ratio increase. They also indicated that convection cells play a crucial role in the segregation mechanism. Elperin and Golshterin ${ }^{16}$ used the particle dynamics method to study the influence of convection on size segregation in a 2D vibration bed. The results showed that the segregation rate increases dramatically as convective rolls occur. Liao et al. ${ }^{17}$ demonstrated that the Brazil nut effect is mitigated by greater intruder surface roughness. They also found that the penetration length of the intruder increases as the intruder surface roughness decreases and that the penetration length scales exponentially with vibration frequency.

Wall friction also has a significant influence on flow behaviors and dynamic properties. Farkas et al. ${ }^{18}$ carried out experiments and simulations to investigate the horizontal transport of granular particles in a vertical vibrating bed with a sawtooth-shaped base. Levanon and Rapaport ${ }^{19}$ investi- $^{-}$ gated the horizontal flow of granular materials in a vertically vibrating sawtooth-shaped base. Their simulation results showed that the induced flow rate varies with bed height and the counter flow could occur at different levels. Hsiau et al. ${ }^{13}$ found that the convection and segregation rates are enhanced with the increase of side wall friction coefficient in a vertical shaker. Hsiau and Yang ${ }^{20}$ reported for a sheared granular flow that rougher wall conditions increased the shear stress and shear rate.

In real granular system, the wall conditions may also be bumpy or uneven. The effect of a bumpy base on the rise dynamics of the intruder and transport properties has not been previously examined and it offers insight into the complexities of segregation. In this study, we performed a series of quasi-2D experiments to investigate the influence of base roughness on the Brazil nut problem and the transport properties. The dependence of the intruder dynamics on the dimensionless vibration acceleration and vibration frequency is also discussed.

\section{EXPERIMENTAL SETUP}

Figure 1 shows the schematic drawing of the experimental apparatus. A Techron VTS-100 electromagnetic vibration system driven by sinusoidal signals produced by a function generator (Meter Inc. DDS FG-503) through a power amplifier (Techron Mode 5530) was used to vertically shake the assembly. The vibration frequency $f$ and the vibration acceleration $a$ were measured by a Dytran 3136A accelerometer fixed to the shaker and connected to an oscilloscope (Tektronix TDS 210). Given the radian frequency $\omega=2 \pi f$, the amplitude $A$ of the vibration is calculated from

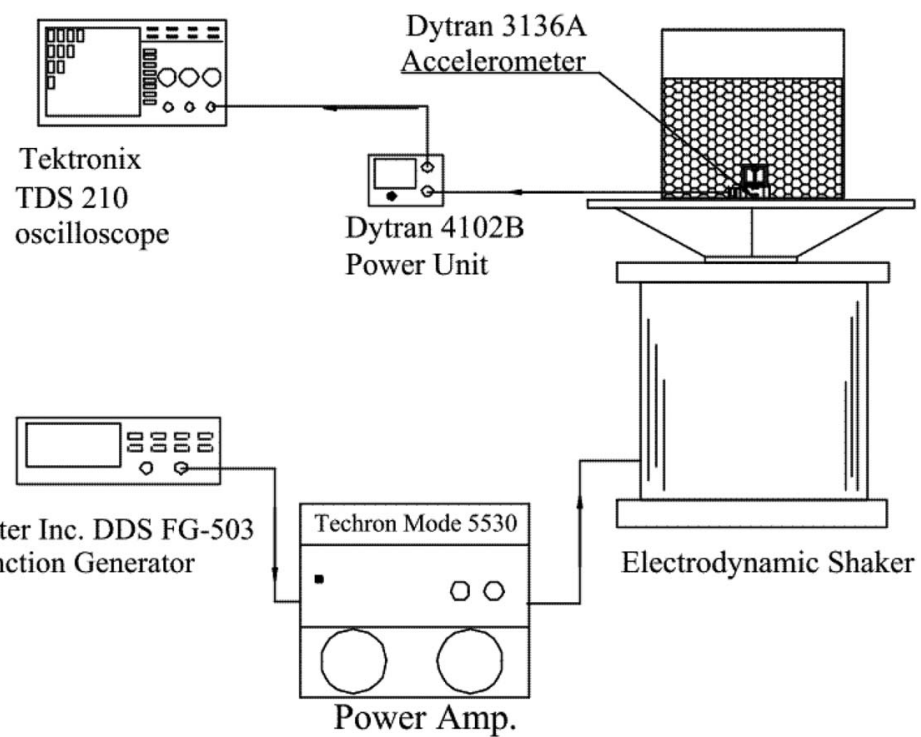

FIG. 1. Schematic drawing of the experimental apparatus. 
(a)

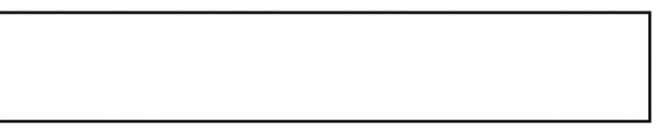

(b)

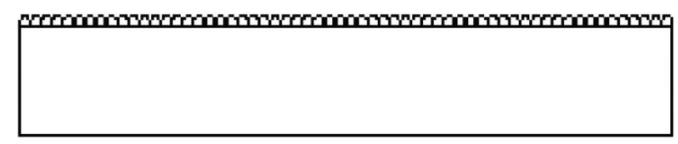

(c)

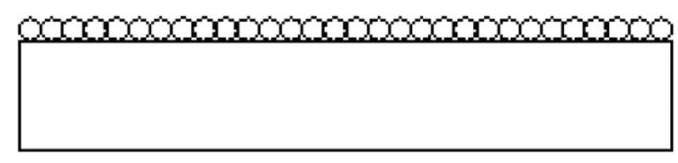

(d)

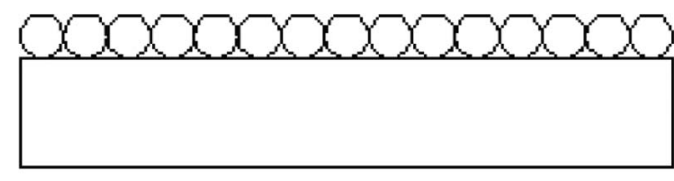

FIG. 2. Illustration the bumpy factor of bottom surface (a) $R_{f}=0$; (b) $R_{f}=0.5$; (c) $R_{f}=1$; (d) $R_{f}=2$.

$A=a / \omega^{2}$. The dimensionless vibration acceleration $\Gamma$ is defined as $\Gamma=a / g$, where $g$ is the gravitational acceleration.

The bed container had glass plates for front and back walls and plexiglass for side and bottom walls. The height, width, and depth of the inside of the container were 150.0, 60.0, and $4.0 \mathrm{~mm}$, respectively. To reduce wall friction, the plates of the tank were carefully cleaned and polished before every experimental test. For the immersed granular material, we used smooth mono-sized glass beads with a diameter of $2 \mathrm{~mm}$ and a density of $2.476 \mathrm{~g} / \mathrm{cm}^{3}$. A cylindrical intruder with a $6.0 \mathrm{~mm}$ diameter and a $3.0 \mathrm{~mm}$ thickness was used in this study. The intruder was made of stainless steel with a density of $7.5 \mathrm{~g} / \mathrm{cm}^{3}$. In this study, we glued a layer of glass beads in a closed packing on the base of container to generate a bumpy base surface (Figure 2).

To quantify the relative size of the particles on the base, we define a base factor $R_{f}$,

$$
R_{f}=\frac{p_{d}}{d_{b}},
$$

where $P_{d}$ is the diameter of glued beads and $d_{b}$ is the diameter of immersed glass beads ( $d_{b}=2 \mathrm{~mm}$ in this study). Four different base factors $\left(R_{f}=0,0.5,1,2\right)$ were used with $R_{f}=0$ for a flat base surface and $R_{f}=2$ for the largest wall protrusions (Table I).

TABLE I. Roughness factor values used in experiments.

\begin{tabular}{lcc}
\hline \hline $\begin{array}{l}\text { Background } \\
\text { particle, } d_{b}(\mathrm{~mm})\end{array}$ & $\begin{array}{c}\text { Particle distance, } \\
p_{d}(\mathrm{~mm})\end{array}$ & Base factor, $R_{f}$ \\
\hline 2 & 0 & 0 \\
1 & 0.5 \\
& 2 & 1 \\
& 4 & 2 \\
\hline \hline
\end{tabular}


For each experimental run, $35 \mathrm{~g}$ of glass beads were poured into the container, with the intruder placed on the center line $3.5 \mathrm{~mm}$ from the bottom. The granular bed was then vertically shaken. In a two-dimensional granular bed, the rougher side-walls induce the stronger particle motions and convection cells. ${ }^{13,17}$ Emery paper (KA961 P60) was glued to the sidewalls of container to generate sufficient shear in the flow field. A high-speed CCD camera (IDT X-3 Plus with grabbing speed of 100 FPS) recorded the front-view of the intruder motion. Using a particle-tracking method with the assistance of an image-processing system, the position of the intruder could be measured.

In addition to measuring the rise dynamics of the intruder, the granular temperature and selfdiffusion coefficient of immersed glass beads were also determined. All images were taken with grabbing speed of 250 FPS after the system had been vibrating for at least 1 min to make sure the flow field was in a steady state. The bed was divided into square bins $(0.3 \mathrm{~cm} \times 0.3 \mathrm{~cm})$, and the autocorrelation technique was employed to process the stored images and to determine the shift of each tracer particle between two consecutive images. From this, the local velocities could be calculated from all the tracer velocities (120-180 tracer particles) occurring in the bin $^{21-25}$

$$
\begin{array}{r}
\left\langle u_{i}\right\rangle=\frac{\sum_{k=1}^{N_{i}} u_{k i}}{N_{i}}, \\
\left\langle v_{i}\right\rangle=\frac{\sum_{k=1}^{N_{i}} v_{k i}}{N_{i}},
\end{array}
$$

where $\left\langle u_{i}\right\rangle$ and $\left\langle v_{i}\right\rangle$ denote the ensemble-average velocities in the horizontal and vertical directions, respectively, in the $i$ th bin with the averaging of velocities from $N_{i}$ tracer particles. The subscript $k$ denotes the $k$ th tracer particle in the $i$ th bin.

The fluctuation velocities in the $i$ th bin were defined as the root mean square of the deviations between the local velocities and the ensemble-average velocities:

$$
\begin{gathered}
\left\langle u_{i}^{\prime 2}\right\rangle^{1 / 2}=\sqrt{\frac{\sum_{k=1}^{N_{i}}\left(u_{k i}-\left\langle u_{i}\right\rangle\right)^{2}}{N_{i}},} \\
\left\langle v_{i}^{\prime 2}\right\rangle^{1 / 2}=\sqrt{\frac{\sum_{k=1}^{N_{i}}\left(v_{k i}-\left\langle v_{i}\right\rangle\right)^{2}}{N_{i}} .}
\end{gathered}
$$

The granular temperature in the $i$ th bin was then determined as

$$
T_{i}=\frac{\left\langle u_{i}^{\prime 2}\right\rangle+\left\langle v_{i}^{\prime 2}\right\rangle}{2} .
$$

The average granular temperature of the whole bed was calculated as

$$
T=\frac{\sum_{i} N_{i} \times T_{i}}{\sum_{i} N_{i}} .
$$

The granular temperature quantifies the fluctuation kinetic energy per unit mass resulting from the random motions of the particles. The granular temperatures were defined as the ensemble average of all tracer particles' fluctuation velocities in a vibrating granular bed, and a higher granular temperature indicates that the vibrating bed is more energetic and fluidized. ${ }^{21,22,25}$

Particle diffusion results from the particle fluctuations. The concept for analyzing the diffusive phenomena of suspended particles undergoing Brownian motion in a liquid was first employed by Einstein. ${ }^{26}$ Campbell $^{27}$ and Savage and Dai ${ }^{28}$ also used this technique to investigate the self-diffusive 
TABLE II. Vibration conditions used in experiments.

\begin{tabular}{lc}
\hline \hline Vibration frequency, $f(\mathrm{~Hz})$ & $\begin{array}{c}\text { Dimensionless vibration } \\
\text { acceleration, } \Gamma\end{array}$ \\
\hline 25 & 3 \\
& 3.6 \\
& 4.2 \\
& 4.8 \\
20 & 5.4 \\
25 & 4.2 \\
30 & \\
35 & \\
40 & \\
\hline \hline
\end{tabular}

behavior of granular flow systems through computer simulation. The self-diffusion coefficient $D_{i j}$ is defined as

$$
\lim _{t \rightarrow \infty}\left\langle\Delta x_{i}(t) \Delta x_{j}(t)\right\rangle=2 D_{i j} t,
$$

where $\Delta x_{i}$ and $\Delta x_{j}$, functions of $t$, represent the diffusive displacements in directions $i$ and $j$ relative to their initial position. Natarajan et al..$^{29}$ and Liao et al. ${ }^{30}$ also employed the similar concept to measure self-diffusion in a vertical channel and in sheared granular flows, respectively.

In this study, we also discuss the influence of convection on the Brazil nut mechanism and the dimensionless strength of the convective flow rate $J$ was measured. ${ }^{13,24,25}$ Hsiau and Chen ${ }^{24}$ indicated that the mass flow rates in the top, right, bottom, and left should be conserved within each convection cell. In the horizontal plane that crosses the center of the convection cells, the horizontal velocity is 0 and only a vertical velocity component $\langle v\rangle_{c}$ exists. Hence at this center location, the convection mass flow rate $\dot{m}$ (with the same horizontal level as the convection centers) can be defined by

$$
\dot{m}=\int_{x=0}^{x=W} \frac{\rho_{p} v\left|\langle v\rangle_{c}\right|}{2} d x
$$

where $v$ is the solid fraction, $\rho_{p}$ is the true bead density, and $W$ is the half width of the container across the convection cell center. Assuming that the solid fraction is constant across each horizontal plane, the channel can be divided horizontally into experimental bins. By this means, $J$ can be expressed as

$$
J=\frac{\dot{m}}{\rho_{p} \nu 2 W \sqrt{g d}_{b}}=\frac{\sum\left|\langle v\rangle_{c} \Delta x\right|}{2 W \sqrt{g d_{b}}},
$$

where $\Delta x$ is the width of each bin. For details of the definition and development, see Hsiau and Chen. $^{24}$

A series of experiments were performed at different vibration conditions. Details of experimental parameters are listed in Table II. Each case was repeated at least five times to calculate the average rise time of the intruder.

\section{RESULTS AND DISCUSSION}

Figure 3 presents the intruder position as a function of time for the four different base factors for fixed $\Gamma=4.2$ and at a vibrational frequency $f=35 \mathrm{~Hz}$. The gradual rise of the intruder from the bottom to the free surface is in all cases because of the Brazil nut effect. ${ }^{4-10}$ The longest rise time occurs for $R_{f}=2$ and the shortest for $R_{f}=0$. In a vertical vibrating bed, external energy is mainly introduced through the inelastic collisions between the particles and the base, and it is 


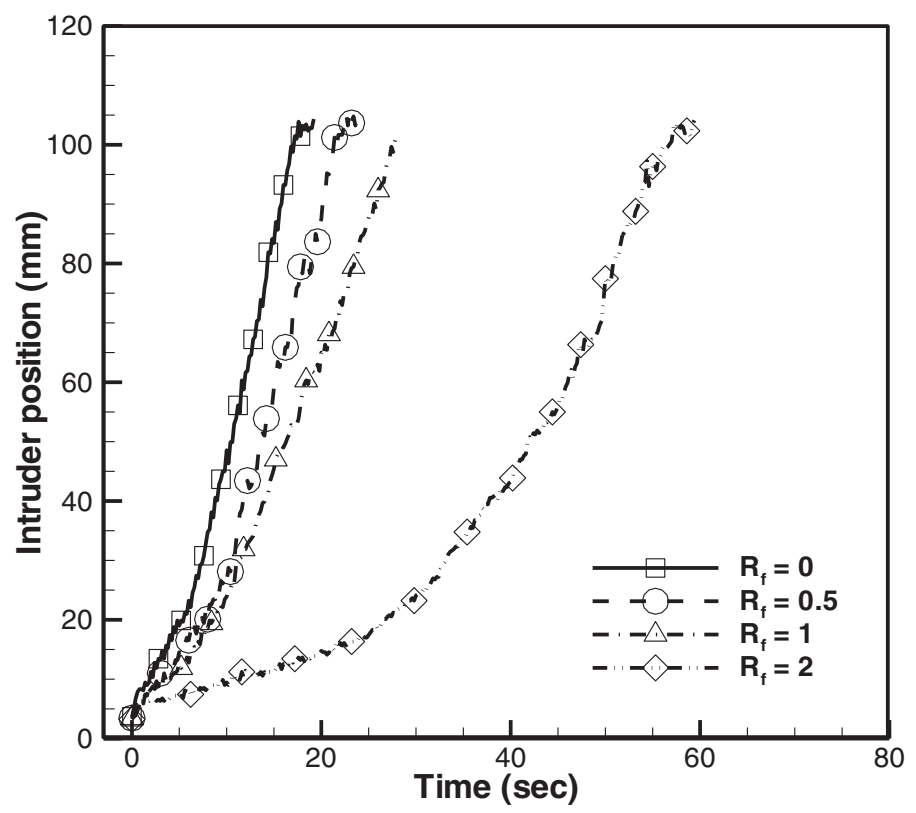

FIG. 3. Intruder position as a function of vibrating time with $R_{f}=0,0.5,1,2$ for fixed $f=25 \mathrm{~Hz}, \Gamma=4.2$.

introduced less effectively into the granular system with the bumpy base surface. The collisions are normal between particles and the bottom wall with a flat bottom wall. With a bumpy base surface, oblique collisions occur between particles and the bottom wall and the surface contact between particle and the base increases due the multiple contact points. The net result is a reduction in the net amount of external energy introduced into the granular system. Consequently, the granular bed receives less net external energy, leading to less particle motion. From previous studies, ${ }^{10,13,17}$ the Brazil nut effect could be minimized because of the weaker fluidization of the granular bed, resulting in the larger resistance between the immersed granular materials and the intruder. For the largest base factor, the net external energy introduced into the system is the smallest resulting in the weaker upward motion and the longest time to reach the free surface, as indicated by the curves in Figure 3.

For this study, the rise time is defined as the time taken by the intruder rising from the initial position ( $3.5 \mathrm{~mm}$ from bottom of the container) to the bed height of $95 \mathrm{~mm}$. Figure 4 shows the rise time plotted as a function of $R_{f}$ with different $\Gamma$ at $f=25 \mathrm{~Hz}$. The rise time increases with $R_{f}$ for each $\Gamma$ because the strength of the particle motions is reduced with the greater base factor. The rise times are also shorter as $\Gamma$ increases for each specific container base condition. Previous research has shown that for higher $\Gamma$, the interactive collision strength is greater and the granular bed becomes more fluidized, enhancing the percolation effect (i.e., stronger Brazil nut effect) and resulting in a faster rise time. ${ }^{2,3,11,17}$ Additionally, the influence of a bumpy base on the rise time is significant at smaller $\Gamma$ and becomes less pronounced at higher $\Gamma$ since more energy can be introduced to the system with higher $\Gamma$. From previous studies, the rise time is similar if the granular bed is fluidized. ${ }^{2,17}$ The better fluidization could be achieved with the higher $\Gamma$. Hence, the rise time increases slightly with the bumpy surface cases at the greater $\Gamma$. On the other hand, the particle motions become weaker and the granular bed becomes less fluidized with the smaller $\Gamma$. Under these conditions, the base roughness has a significant influence on the dynamic properties and leads to the larger variation of rise time for different base conditions.

In the lower part of the granular bed, particle motions and interactive collisions are weaker and the bed concentration is denser; hence, inertial forces then dominate the Brazil nut effect. ${ }^{14,15,17}$ Nahmad-Molinari et al. ${ }^{14}$ defined the penetration length $P_{l}$, to quantify the distance that the intruder penetrates the granular bed in each cycle. Because the kinetic energy of the intruder is dissipated by frictional drag as it penetrates the granular bed. They derived a model based on the simple energy 


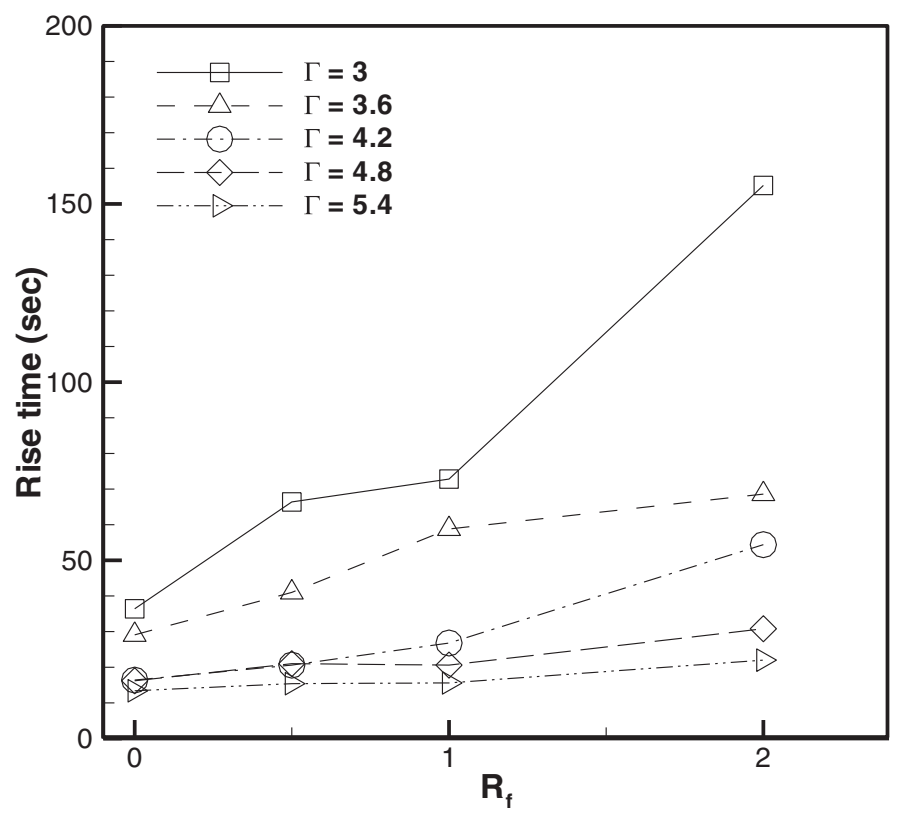

FIG. 4. Rise time as a function of $R_{f}$ with different $\Gamma$ for fixed $f=25 \mathrm{~Hz}$.

balance per cycle ${ }^{14}$

$$
\frac{1}{2} m_{i} v_{t}^{2}=\beta P_{l},
$$

where $m_{i}$ is the intruder mass, $v_{t}$ is the intruder take-off velocity as the system reaches a negative acceleration, $a=-g, \beta$ is the drag force between beads and intruder, and $P_{l}$ is the penetration length. $v_{t}$ is the value of $\dot{z}(t)$ when $\ddot{z}(t)=-g$, and $z(t)=A \sin (\omega t)$. Hence,

$$
v_{t}=\left[A^{2} \omega^{2}-g^{2} / \omega^{2}\right]^{1 / 2} .
$$

The intruder position with different base factors is plotted in Figure 5 as a function of vibrating cycle $(t \omega / 2 \pi)$ at $f=25 \mathrm{~Hz}, \Gamma=4.2$. The slope of each curve is the penetration length per cycle of the corresponding intruder, but not the velocity. ${ }^{14}$ Only the lower parts of the curves as shown in Fig. 3 have linear fits, owing to the reduction in convection that occurs in the upper part of bed.

From linear fits to the data, we can determine the penetration length per vibration cycle for different base factors, ${ }^{14}$ as plotted as a function of vibration amplitude in Figure 6. As shown, the penetration length decreases with greater base factor at the same vibration condition. The penetration length also becomes larger as the vibration amplitude increases. According to Eq. (11), the penetration length is inversely proportional to drag force $\beta$. A greater base factor results in weaker particle fluidization and a denser packing structure, and increases the drag force between the immersed glass beads and the intruder, yielding a shorter penetration length.

To test this physical argument, we calculated $\beta$ according to Eq. (11). Figure 7 shows $\beta$ plotted against $\Gamma$ with different base factors; $\beta$ is observed to increase with greater base factor at identical $\Gamma$. The curves also show that $\beta$ is reduced as $\Gamma$ increases. Convection and fluidization are both enhanced as $\Gamma$ is increased and the flow behavior of the granular materials changes from a quasi-static state to a dynamic state. Therefore, the drag force acting on the intruder is smaller. Additionally, with different base factors, an increase in $\Gamma$ reduces both the drag force $\beta$ and the variation of $\beta$ with different $R_{f}$.

In this study, we also investigated the rise dynamics of the intruder with various vibration frequencies $f$. Figure 8 shows the intruder position for different base factors plotted as a function of time for $f=25$ and $35 \mathrm{~Hz}$ at $\Gamma=4.2$. Similarly, the intruder with the smallest base factor rises fastest to the surface of the granular bed for each frequency. At $35 \mathrm{~Hz}$, a two-stage rising process of 


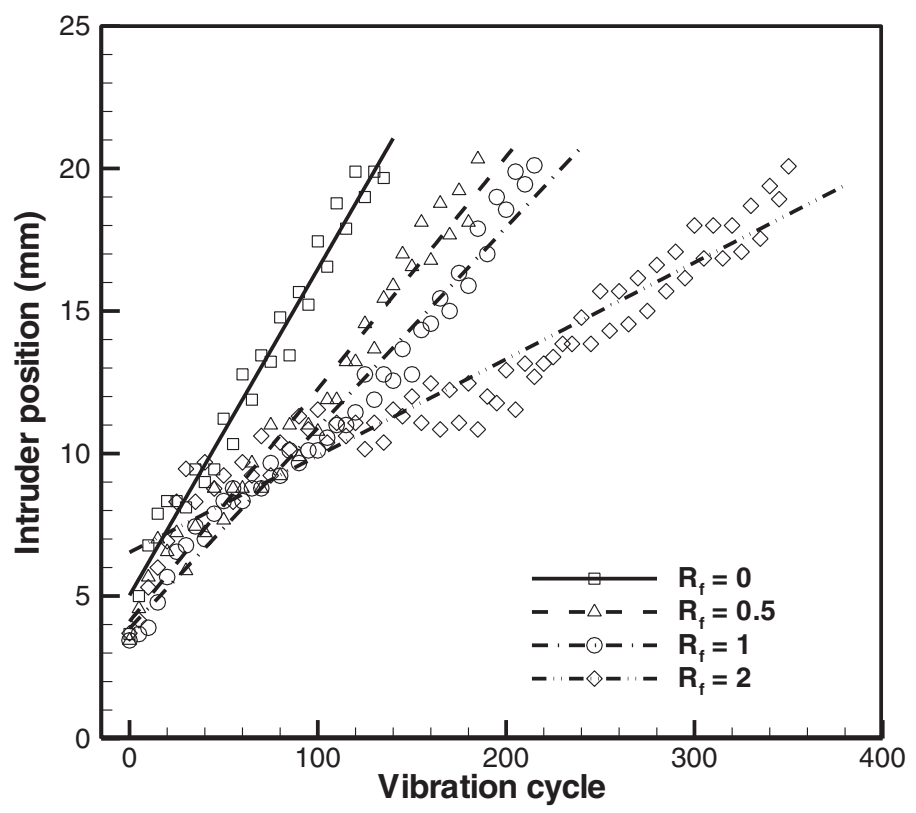

FIG. 5. Intruder position as a function of vibrating cycle (tw/2 $\pi$ ) with $f=25 \mathrm{~Hz}, \Gamma=4.2$.

the intruder is observed. The intruder rises slowly in the lower part of bed but then rises more rapidly in the upper part of granular bed due to convection. ${ }^{17}$ At the higher vibration frequency, the lower part of the granular bed is relatively denser because of the weaker particle motion, resulting in the greater drag force between immersed glass beads and the intruder and lower intruder mobility. ${ }^{17,24}$ Therefore, the intruder experiences difficulty rising to the surface. A quasi-linear rise dynamic is also observed at the smaller vibration frequencies; there, convection is stronger and the whole bed is more fluidized. ${ }^{24}$ Under this condition, the intruder has higher mobility and rises easily. An interesting result is also found that the intruder height does not change with time for the greatest base factor

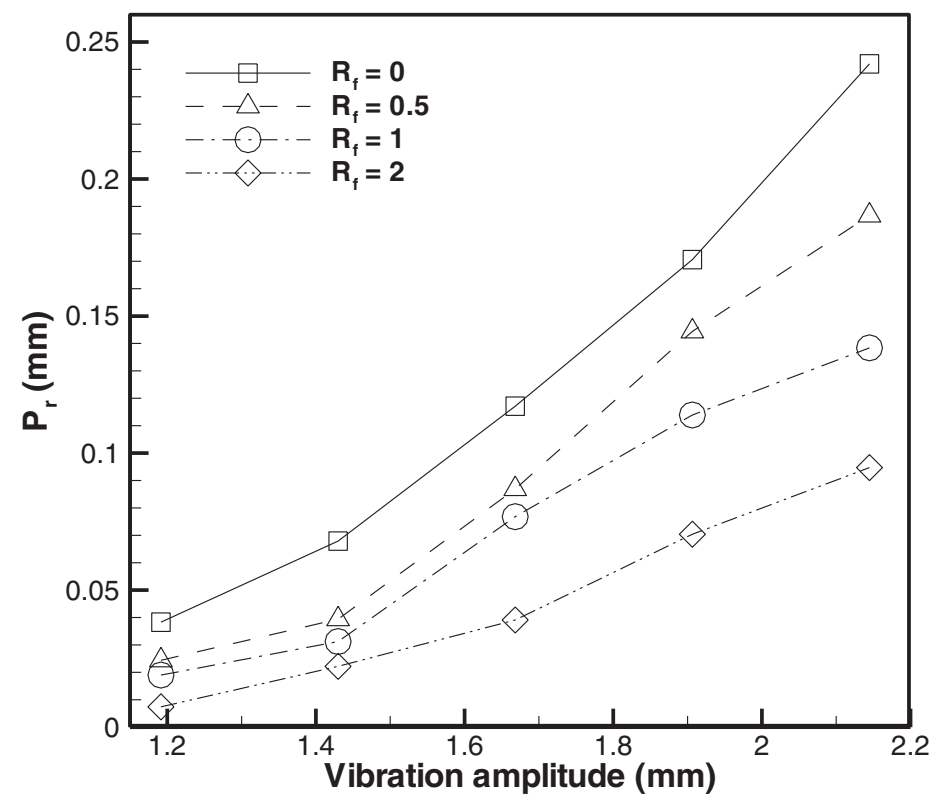

FIG. 6. Penetration length of per vibration cycle as a function of vibration amplitude with different $R_{f}$ for fixed $f=25 \mathrm{~Hz}$. 


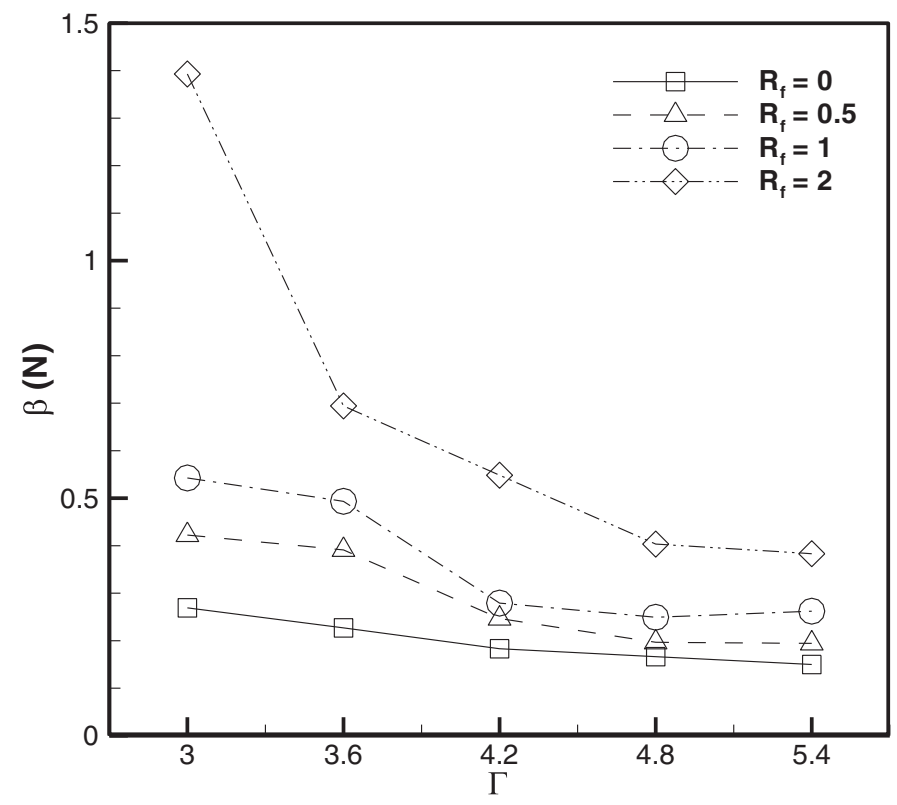

FIG. 7. Friction drag force $\beta$ plotted as a function of $\Gamma$ with different $R_{f}$ for fixed $f=25 \mathrm{~Hz}$.

$\left(R_{f}=2\right)$ with $f=35 \mathrm{~Hz}$ and $\Gamma=4.2$. The result supports the argument that the external energy could not be introduced into the granular system effectively, resulting in the weak fluidization of bed with the greatest bumpy base. Consequently, the solid-like region is formed with larger resistance between the immersed beads and the intruder in the lower part of bed. Therefore, the Brazil nut effect is absent as shown in Figure 8.

The variation of rise time with vibration frequency for the different base factors at $\Gamma=4.2$ shows that the rise times are lengthened with the higher vibration frequencies (Figure 9). The results also indicate that the variation in rise time with different base factors increases with the vibration

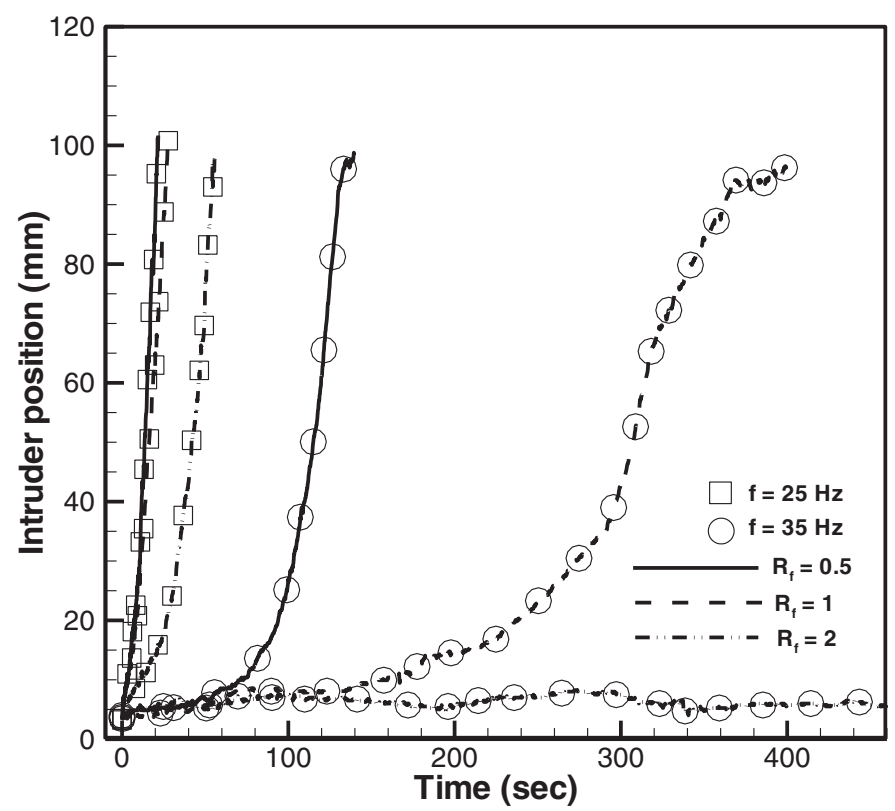

FIG. 8. Intruder position for intruders of different $R_{f}$ as a function of vibrating time with $f=25,35$, and $\Gamma=4.2$. 


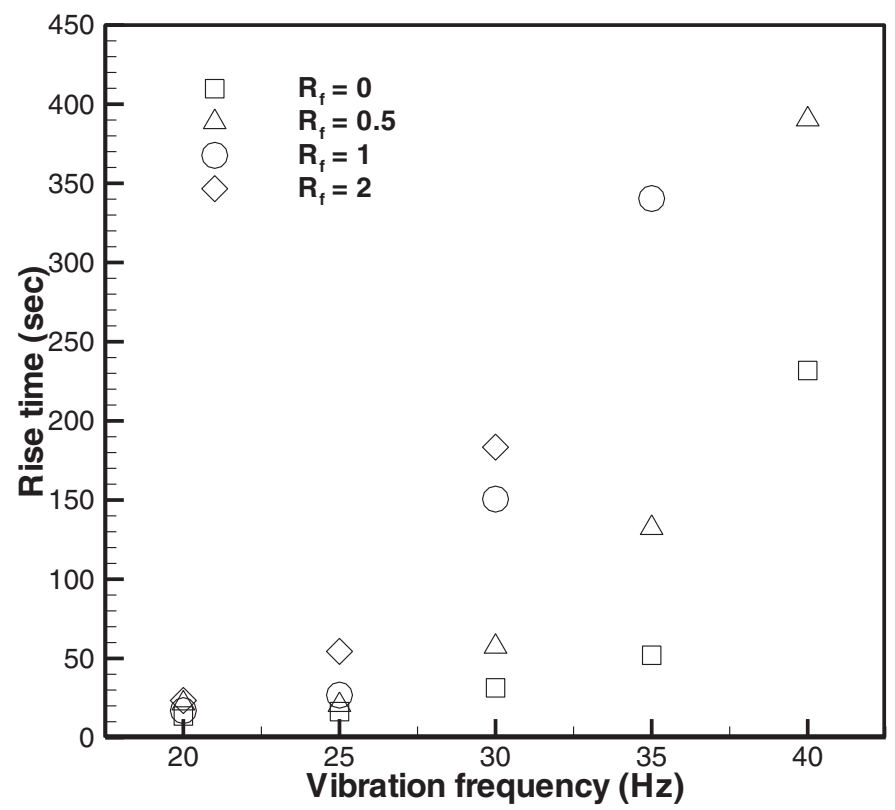

FIG. 9. Rise time as a function of vibration frequency with different $R_{f}$ for fixed $\Gamma=4.2$.

frequency. Some data points are not shown in Figure 9 because the Brazil nut effect is absent for those cases. For each base condition at smaller vibration frequencies the granular bed is better fluidized, resulting in the stronger particle motions and interactive collisions, resulting in the smaller variation in the rise time with different base factors. Conversely, at higher vibration frequencies, the flow within the granular bed displays weaker fluidization and particle motions. ${ }^{10,17,24}$ Additionally, the net external energy introduced into the granular bed is reduced and further weakens particle motions as the base factor is increased, leading to the significant variation of rise time with different base factors.

The variation in penetration length with base factor (Figure 10) for five different vibration frequencies indicates a weakened penetration as base factors increases in each specific vibration frequency case. The physical arguments are similar to the preceding discussions. The results are also in accord with the way the intruder rise time is larger at higher vibration frequencies.

Figure 11 presents the drag force $\beta$ with different vibration frequencies against base factor, and shows an enhancement with increasing base factor. The effective external energy introduced into the granular bed decreases with the increasing base factor, resulting in the less fluidized granular materials. Hence, the drag force is enhanced with the larger base factor. It is noted that some data points are not shown in Figure 11 because the brazil-nut phenomenon does not occur in those cases. To maintain a constant dimensionless acceleration, the amplitude increases as the frequency decreases. Hence, the drag force is smaller and the base factor does not have a significant influence on drag at the lowest vibration frequency because of the better fluidization in the granular bed $(f=20 \mathrm{~Hz})$.

The transport properties of immerse glass beads plays a crucial role in the Brazil nut mechanism. In the following, we discuss the Brazil-nut problem from micromechanics by measuring the transport properties of immersed glass beads. Figure 12 shows the average granular temperature and rise time as a function of $R_{f}$ for fixed $f=25 \mathrm{~Hz}$ and $\Gamma=3$. The base condition has a significant influence on the granular temperature. The average granular temperature decreases and the rise time increases as the base factor is enhanced. The total external energy is the same for fixed vibration condition. More effective energy could be introduced into the bed to increase the granular temperature with the smaller base factor. The particle motions and interactive collisions become stronger and the bed has better fluidization as the granular temperature is larger. Hence, the intruder experiences 


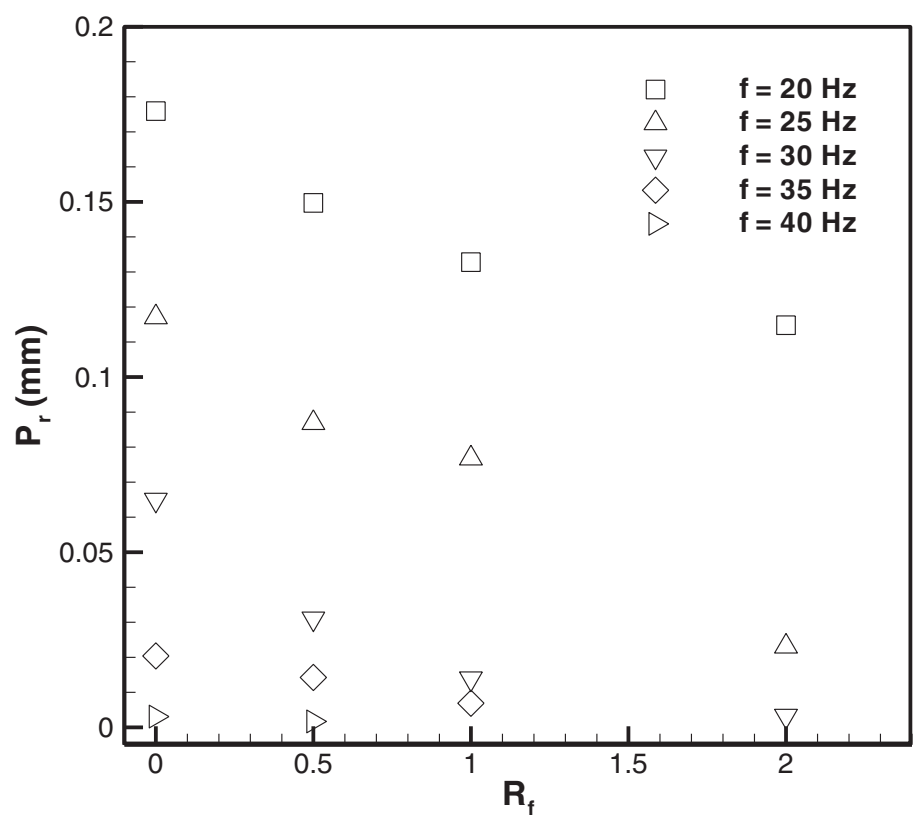

FIG. 10. Penetration length versus $R_{f}$ with different vibration frequency for fixed $\Gamma=4.2$.

less resistance during the rise process and leads to the shorter rise time with the larger granular temperature.

The particle diffusion is an important parameter to study in the mixing and segregation of granular materials. The self-diffusion coefficients are determined from the relation between meansquare diffusive displacements and time, as shown in Eq. (8) using similar experimental procedures as found in previous studies. ${ }^{21,22,30}$ Self-diffusion coefficients and rise time are plotted as a function of $R_{f}$ for fixed $f=25 \mathrm{~Hz}$ and $\Gamma=3$ as shown in Figure 13. The self-diffusion coefficients are inversely proportional to rise time with the increase of base factor. Particles diffusive behavior results from

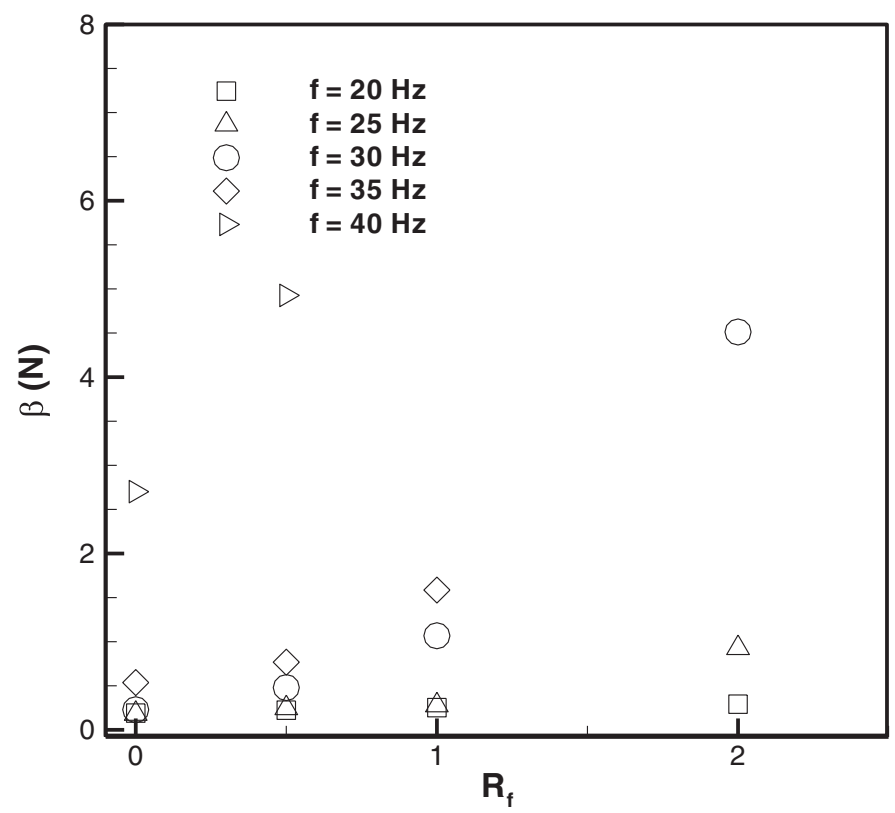

FIG. 11. Friction drag force $\beta$ plotted as a function of $R_{f}$ with different vibration frequency. 


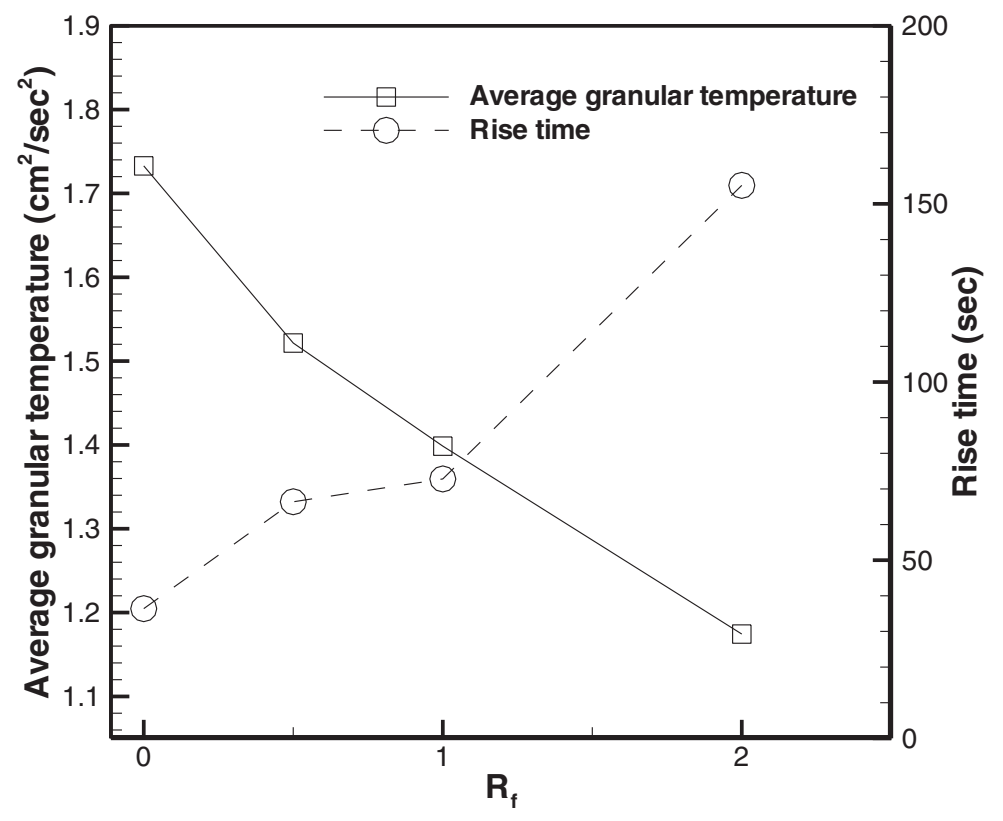

FIG. 12. Average granular temperature and rise time as a function of $R_{f}$ for fixed $f=25 \mathrm{~Hz}$ and $\Gamma=3$.

the particle fluctuations. Previous studies also ${ }^{28,31}$ indicated that the self-diffusion coefficient is proportional to the square root of $T$. For the greater base factor, the external energy could not introduced into system effectively, leading to the weaker interactive collisions and the smaller granular temperature. Therefore, the self-diffusion coefficients decrease and cause the longer rise time with the increase of base factor as shown in Figure 13.

Figure 14 shows the dimensionless convection flow rate $J$ and rise time plotted as a function of $R_{f}$ for fixed $f=25 \mathrm{~Hz}$ and $\Gamma=3$. It could be found that $J$ increases and rise time decreases with the decreasing of base factor. The flow field is more fluidized, which strengthens the convection with

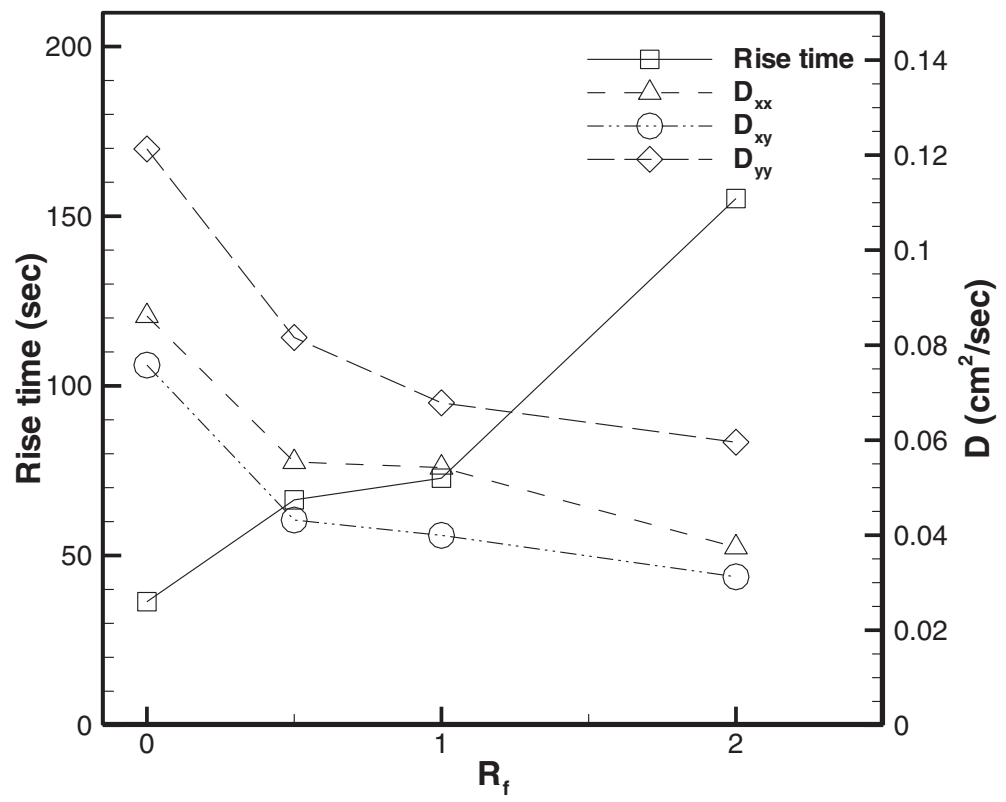

FIG. 13. Self-diffusion coefficients $D$ and rise time as a function of $R_{f}$ for fixed $f=25 \mathrm{~Hz}$ and $\Gamma=3$. 


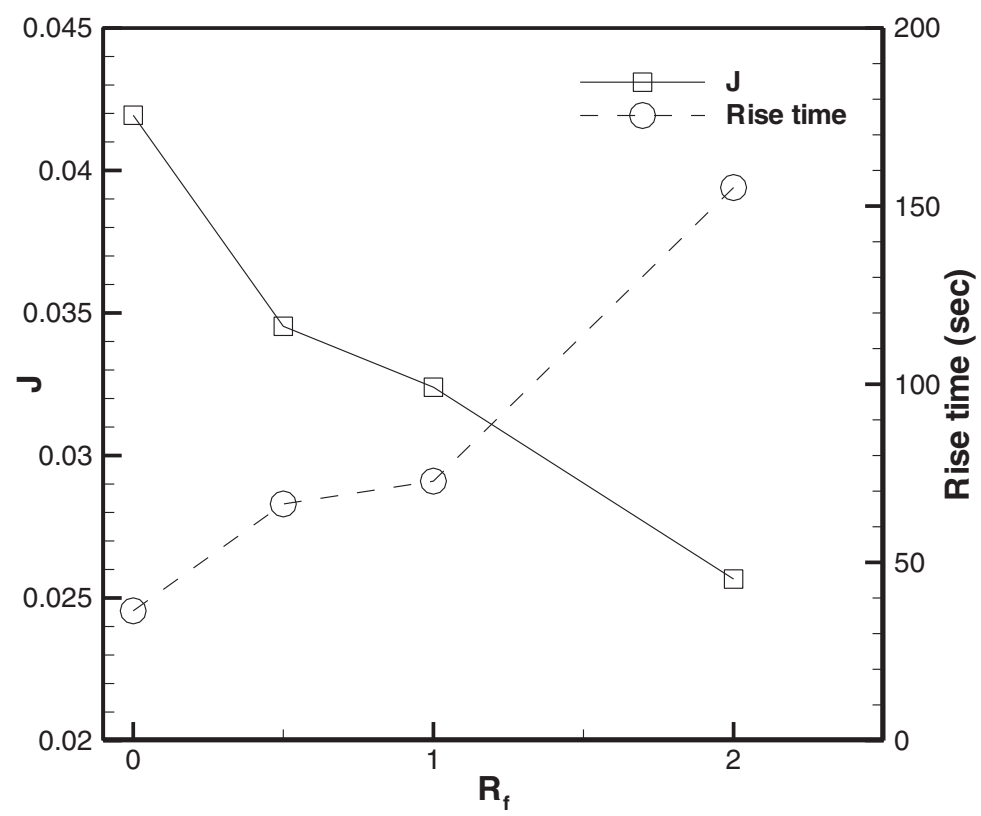

FIG. 14. Dimensionless convection flow rate $J$ and rise time as a function of $R_{f}$ for fixed $f=25 \mathrm{~Hz}$ and $\Gamma=3$.

the smaller base factor. As a result, $J$ is enhanced and rise time is decreased with the decrease of base factor. The result is in agreement with the previous studies. ${ }^{13,17}$

\section{CONCLUSION}

A series of experiments are carried out to investigate the influence of surface roughness of the container base on the Brazil nut problem in a quasi-2D vertical vibration granular bed. The motion of the intruder was recorded using a high-speed camera and an analysis of intruder rise dynamics was presented and discussed. The results show that the intruder rise dynamics and transport properties of the immersed glass beads are influenced significantly by the base roughness. Furthermore, the Brazil nut effect is mitigated, yielding longer rise times with the greater base factor. The penetration length increases as the base factor decreases. Additionally, the drag force, as calculated according to the theory, is enhanced as the base factor increases. Finally, we find that the granular temperature, diffusion coefficients and dimensionless convection flow rate are reduced with the larger base factor and these quantities are inversely proportional to the rise time. The results are contrary to the most previous studies where the roughness of a wall surface enhances the dynamic properties and flow behavior. This paper demonstrates that the base roughness impacts the flow differently than wall friction and diminishes the overall transport within the granular system.

\section{ACKNOWLEDGMENTS}

The authors acknowledge the support from the National Science Council, Taiwan (R.O.C.) for this research (Grant Nos. NSC 100-2221-E-008-078-MY3 and NSC 101-3113-E-008-004).

${ }^{1}$ J. Duran, J. Rajchenbach, and E. Clément, “Arching effect model for particle size segregation,” Phys. Rev. Lett. 70, 2431 (1993).

${ }^{2}$ W. Cooke, S. Warr, J. M. Huntley, and R. C. Ball, "Particle size segregation in a two-dimensional bed undergoing vertical vibration,” Phys. Rev. E 53, 2812 (1996).

${ }^{3}$ J. B. Knight, H. M. Jaeger, and S. R. Nagel, "Vibration-induced size separation in granular media: The convection connection," Phys. Rev. Lett. 70, 3728 (1993).

${ }^{4}$ A. Rosato, K. J. Strandburg, F. Prinz, and R. H. Swendsen, "Why the Brazil nuts are on top: Size segregation of particulate matter by shaking," Phys. Rev. Lett. 58, 1038 (1987). 
${ }^{5}$ L. Vanel, A. D. Rosato, and R. N. Dave, "Rise-time regimes of a large sphere in vibrated bulk solids," Phys. Rev. Lett. 78, 1255 (1997).

${ }^{6}$ A. P. J. Breu, H. M. Ensner, C. A. Kruelle, and I. Rehberg, "Reversing the Brazil-nut effect: Competition between percolation and condensation," Phys. Rev. Lett. 90, 014302 (2003).

${ }^{7}$ M. E. Mobius, X. Cheng, G. S. Karczmar, S. R. Nagel, and H. M. Jaeger, "Intruders in the dust: Air-driven granular size separation,” Phys. Rev. Lett. 93, 198001 (2004).

${ }^{8}$ C. C. Liao, S. S. Hsiau, T. H. Tsai, and C. H. Tai, "Segregation to mixing in wet granular matter under vibration," Chem. Eng. Sci. 65, 1109 (2010).

${ }^{9}$ R. Brito, H. Enríquez, S. Godoy, and R. Soto, "Competition of Brazil nut effect, buoyancy, and inelasticity induced segregation in a granular mixture," Phys. Rev. E 77, 061301 (2008).

${ }^{10}$ S. S. Hsiau and H. Y. Yu, "Segregation phenomena in a shaker," Powder Technol. 93, 83 (1997).

${ }^{11}$ A. Saez, F. Vivanco, and F. Melo, "Size segregation, convection, and arching effect," Phys. Rev. E 72, 021307 (2005).

${ }^{12}$ M. P. Ciamarra, M. D. De Vizia, A. Fierro, M. Tarzia, A. Coniglio, and M. Nicodemi, "Granular species segregation under vertical tapping: Effects of size, density, friction, and shaking amplitude," Phys. Rev. Lett. 96, 058001 (2006).

${ }^{13}$ S. S. Hsiau, P. C. Wang, and C. H. Tai, "Convection cells and segregation in a vibrated granular bed," AIChE J. 48, 1430 (2002).

${ }^{14}$ Y. Nahmad-Molinari, G. Canul-Chay, and J. C. Ruiz-Suarez, "Inertia in the Brazil nut problem," Phys. Rev. E 68, 041301 (2003).

${ }^{15}$ D. A. Huerta and J. C. Ruiz-Suarez, "Vibration-induced granular segregation: A phenomenon driven by three mechanisms," Phys. Rev. Lett. 92, 114301 (2004).

${ }^{16}$ T. Elperin and E. Golshtein, "Effects of convection and on size segregation in vibration in vibrated granular beds," Physica A 247, 67 (1997).

${ }^{17}$ C. C. Liao, S. S. Hsiau, and C. S. Wu, "Experimental study on the effect of surface roughness on Brazil nut problem in a vertically vibrated bed," Phys. Rev. E 86, 061316 (2012).

${ }^{18}$ Z. Farkas, P. Tegzes, A. Vukics, and T. Vicsek, "Transitions in the horizontal transport of vertically vibrated granular layers," Phys. Rev. E 60, 7022 (1999).

${ }^{19}$ M. Levanon and D. C. Rapaport, "Stratified horizontal flow in vertically vibrated granular layers," Phys. Rev. E 60 011304 (2001).

${ }^{20}$ S. S. Hsiau and W. L. Yang, "Stresses and transport phenomena in sheared granular flows," Phys. Fluids 14, 612 (2002).

${ }^{21}$ S. S. Hsiau and Y. M. Shieh, "Fluctuations and self-diffusion of sheared granular material flows," J. Rheol. 43, 1049 (1999).

${ }^{22}$ C. C. Liao and S. S. Hsiau, "Influence of interstitial fluid viscosity on transport phenomenon in sheared granular materials," Chem. Eng. Sci. 64, 2562 (2009).

${ }^{23}$ C. C. Liao, S. S. Hsiau, and To Kiwing, "Granular dynamics of a slurry in a rotating drum," Phys. Rev. E 82, 010302 (2010).

${ }^{24}$ S. S. Hsiau and C. H. Chen, "Granular convection cells in a vertical shaker," Powder Technol. 111, 210 (2000).

${ }^{25}$ S. S. Hsiau, C. C. Liao, P. Y. Sheng, and S. C. Tai, "Experimental study the influence of bed height on convection cell formation," Exp. Fluids 51, 795 (2011).

${ }^{26}$ A. Einstein, Investigations on the Theory of Non-Uniform Gases (Dover Publ. Co., New York, 1956), Chap. 1, p. 12.

${ }^{27}$ C. S. Campbell, "Self-diffusion in granular shear flows," J. Fluid Mech. 348, 85 (1997).

${ }^{28}$ S. B. Savage and R. Dai, "Studies of granular shear flows: Wall slip velocities, 'layering' and self-diffusion,” Mech. Mater. 16, 225 (1993)

${ }^{29}$ V. R. Natarajan, M. L. Hunt, and E. D. Taylor, "Local measurements of velocity fluctuations and diffusion coefficients for a granular material flow," J. Fluid Mech. 204, 1 (1995).

${ }^{30}$ C. C. Liao, S. S. Hsiau, J. S. Li, and C. H. Tai, "The influence of gravity on dynamic properties in sheared granular flows," Chem. Eng. Sci. 65, 2531 (2010).

${ }^{31}$ S. S. Hsiau and M. L. Hunt, "Kinetic theory analysis of flow-induced particle diffusion and thermal conduction in granular material flows," Trans. ASME C: J. Heat Transf. 155, 541 (1993). 